\title{
Jugend, Verwundbarkeit und Soziale Diskriminierung Lebenschancen und Konfliktpotentiale Ländlicher Jugendlicher in Sri Lanka
}

\section{Markus Mayer, Colombo}

Jugendliche haben bislang weder in der wissenschaftlichen noch entwicklungspolitischen Diskussion um Verwundbarkeit ausreichend Beachtung als eine eigenständige Zielgruppe erfahren. Insbesondere bei Fragen der Lebenssicherung («livelihood security») im Kontext gesellschaftlicher Risiken stellt Jugend aber eine wichtige Referenzgruppe dar, anhand derer nicht nur Aspekte der (Über-) Lebenssicherung analysiert, sondern darüber hinaus gesellschaftliche und soziale Konfliktpotentiale eingeschätzt werden können.

Dabei wird die These vertreten, daß sich Formen der sozialen Diskriminierung in besonderer Weise auf Jugendliche auswirken, die sich noch im Prozeß der Identitätsbildung und auf der Suche nach einer adäquaten gesellschaflichen Position befinden. Lebenssicherung weist für Jugendliche daher oftmals eine sehr viel stärkere perspektivische Dimension auf: nicht alleine die unmittelbare Lebenssicherung steht hier im Mittelpunkt, sondern vor allem die Frage nach ausreichender Chancengleichheit, bestimmte Lebensziele realistischerweise erreichen zu können. Jugend stellt dann nicht nur eine Altersgruppe dar, die am stärksten von gesellschafts-politischen Diskriminierungsprozeßen betroffen ist, sondern vielfach auch am leichtesten zu radikalisieren ist, wenn ihre Bedürfnisse nicht ausreichend adressiert werden.

Es geht also nicht nur darum, gewalttätige Konflikte als ein gesellschaftliches Risiko anzusehen, welches die Überlebenssicherung verwundbarer Gruppen erschwert, sondern auch Diskriminierungsprozeße, insbesondere wenn auf Jugendliche ausgerichtet, als eine Gefahr anzusehen, eben jenes Risiko gewalttätiger Konflikte in einer Gesellschaft zu erhöhen. In diesem Beitrag soll der Verwundbarkeitsansatz daher konzeptionell auch für die Einschätzung solcher Konfliktpotentiale eingesetzt werden.

Insbesondere für den entwicklungspolitischen Kontext kommt dieser Frage eine hohe Bedeutung zu, da es in jüngster Zeit zu einer intensiven Diskussion gekommen ist, inwiefern Entwicklungszusammenarbeit über die Zielsetzung der Armutsbekämpfung hinaus auch einen Beitrag zur Konfliktprävention leisten kann. Sicherheitspolitik und Entwicklungspolitik - so die Forderung - sollen enger miteinander verknüpft werden, um dadurch zum einen eine nachhaltigere Entwicklung zu ermöglichen, zum anderen aber auch die ökonomischen und sozialen Folgekosten von Konflikten zu vermeiden. Über die politische Neuformulierung der Rolle der Entwicklungszusammenarbeit ist es mittlerweile sowohl innerhalb der praxisorientierten Organisationen wie auch innerhalb der Wissenschaft zu ersten konzeptionellen Überlegungen gekommen. Dabei ist allerdings festzuhalten, daß der theoretische Diskurs über Konfliktprävention im Moment weiter entwickelt ist als dessen Umsetzung in der Praxis $(E+Z$ 2000).

Einen guten Überblick über den Stand der Diskussion gibt das Themenheft von Entwicklung und Zusammenarbeit (E+Z 2000) zur Krisenprävention und Konfliktbearbeitung. Eine grundlegende Einführung in die Zusammenhänge zwischen Konflikt und Entwicklungszusammenarbeit gibt ANDERSON (1999). In Deutschland sind jüngst zwei wichtige Publikationen erschienen, die einen sehr guten Überblick über den Stand der Diskussion innerhalb der entwicklungspolitischen Organisationen geben (FAHRENHORST 2000 sowie Mehler \& Ribaux 2000). Eine kritische Rezeption der Potentiale und Grenzen möglicher Beiträge von Projekten zur Konfliktlösung auf der Mikround Meso-Ebene unter Bezug auf Sri Lanka findet sich bei Bauer, Bigdon \& Korf (2000). Anhand der Zusammenhänge zwischen Jugend, Verwundbarkeit und Konflikten möchte der folgende Beitrag einige konzeptionelle und empirische Anregungen liefern, das Verwundbarkeitskonzept als ein Instrument der Konfliktanalyse für die Entwicklungsplanung einzuführen.

\section{Jugend als Untersuchungsgegenstand}

Die Wahrnehmung von Jugend als einer besonders verwundbaren gesellschaftlichen Gruppe bezieht sich insbesondere im Kontext der Entwicklungsländer häufiger auf Kinder als auf Jugendliche (Roche \& TUCKER 1997: 6). Jugend alleine über eine bestimmte Alterspanne definieren zu wollen, greift aber in jedem Falle zu kurz. Die Lebenssituationen Jugendlicher in einer bestimmten Gesellschaft werden außer in der gleichen Alterskategorie i.d.R. kaum Überschneidungen oder gar Ähnlichkeiten aufweisen (RUDOLPH 1997: 18). Weniger als vom Alter wird das Verhalten Jugendlicher in viel stärkeren Ausmaße von Klassen- und Schichtzugehörigkeit geprägt und von Kategorien wie 
Geschlecht, Bildungsstand, Erziehung etc. Diese Kategorien können weitaus stärker differenzierend wirken als die auf Alter beruhenden Unterschiede zwischen Jugendlichen und Erwachsenen (Rucht \& Rотн 2000: 284).

Die reine Altersdefinition wird dabei insbesondere zur Rechtfertigung bestimmter politischer Interventionen verwendet, die sich gezielt auf Jugendliche beziehen sollen. Mit Jugend wird dabei oft eine «systemsprengende Haltung», "politischer Radikalismus» und «Unangepaßtheit» assoziiert. Gesellschaftliche Protestbewegungen der Jugend werden in der breiten Öffentlichkeit dann schnell als ein Generationskonflikt wahrgenommen (Rотн \& Ruснт 2000: 11). Kritisch an dieser Suggestion, daß Jugendliche sich automatisch in einem Spannungsfeld zwischen Rebellion und Anpassung befinden, ist die gängige Schlußfolgerung, daß Jugendliche eine altersbedingte Übergangsphase durchlaufen, auf die sich ihre Schwierigkeiten mit den bestehenden Verhältnissen zurückführen lassen. Damit verbindet sich die Möglichkeit, allgemeine gesellschaftliche Mißstände als ein Generationsproblem zu klassifizieren und Hilfeleistungen für Jugendliche anzufordern (durch Sozialarbeiter, Lehrer etc.), anstatt gesellschaftliche Probleme (wie z.B. Klassenstrukturen) anzusprechen (COHEN 1997: 192).

Jugend stellt aber in erster Linie eine Phase der Sozialisierung und der Identitätsbildung in historischer und kulturspezifischer Weise dar und weniger einen bestimmten Entwicklungsstand per se (WYN \& DWYER 1999: 14). Um diesem Prozeßcharakter von Jugend gerecht zu werden, muß sich die Analyse der Situation und Probleme Jugendlicher zum einen mit den Interaktionen innerhalb der Familie verknüpfen, zum anderen mit sich wandelnden sozialen und ökonomischen Rahmenbedingungen und damit mit der Gesellschaft als Ganzem (COHEN 1997: 220f.).

Die Forschung hat also dabei sowohl die individuellen, biographischen Dimensionen von Jugend zu berücksichtigen, wie auch die strukturellen Rahmenbedingungen (in bezug auf gesellschaftliche, ökonomische, politische und kulturelle Formationen). Auch der unterschiedliche Zugang zu Ressourcen (materieller wie immaterieller Natur) ist zu analysieren, da sich darüber zahlreiche Formen der sozialen Benachteiligung verbinden, wie z.B. die vielfältige Diskriminierung von Mädchen im Vergleich zu Jungen (Rudolph 1997: 69). Besonderes Augenmerk muß aber auch darauf verwandt werden, die Jugendlichen selbst stärker in den Forschungsprozeß miteinzubeziehen, womit innerhalb der Jugendforschung der Bedarf nach qualitativen und partizipativen Forschungsmethoden wächst.

\section{Jugend und Konflikte in Sri Lanka}

Sri Lanka's Geschichte und Gegenwart ist gekennzeichnet von gewalttätigen Konflikten, die vornehmlich von gesellschaftlich marginalisierten Jugendlichen gegen das etablierte Regierungssystem ausgetragen werden. Nicht von ungefähr gelten Jugendliche daher auch als eine besonders kritische Zielgruppe im Kontext sri lankischer Armutsbekämpfungsprogramme. Der sri lankische Staat hat insbesondere eine Reihe von ambitiösen Regionalentwicklungsvorhaben für den Süden des Landes initiiert, um erneuten gewaltsamen Jugendkonflikten entgegenzuwirken (MAYER 2000).

Der prominenteste Konflikt stellt die gewaltsame Auseinandersetzung zwischen der sri lankischen, von Sinhalesen dominierten Regierung und der tamilischen Minderheit im Norden und Osten des Landes dar, ein Konflikt der vor allem zwischen der sri lankischen Armee und der tamilischen Sezession-Bewegung «Liberation Tigers of Tamil Eelam» (LTTE) geführt wird und nach konservativen Schätzungen bis zu 60.000 Menschen das Leben gekostet und bis zu 800.000 Vertriebenen geführt hat (MARGA 2001).

Daneben sah sich Sri Lanka aber auch zweimal mit einem Aufstand gebildeter jugendlicher Sinhalesen aus den ländlichen Landesteilen des Südens konfrontiert, der vor allem Ende der 80er Jahre das Land an den Rande des Zusammenbruches brachte. Die Niederschlagung dieser Jugendaufstände kostete schätzungsweise weiteren 60.000 , vornehmlich jugendlichen Menschen das Leben (Seneratne 1997).

Auch zu Beginn des neuen Jahrhunderts erscheint Sri Lanka am Scheideweg zu stehen: der Bürgerkrieg im Norden und Osten des Landes hat deutlich an Schärfe zugenommen, zudem hat die politisch motivierte Gewalt zwischen den beiden großen sinhalesischen Parteien im Vorfeld der jüngsten Wahlen eine immer drastischere Dimension angenommen, und im Hochland und an der Ostküste zeichnet sich die Entstehung neuer Konfliktherde mit den indischen Plantagentamilen und Muslims ab. Zudem konnte in den letzten zwei Jahren die radikale «Janata Vimukti Peramuna» (JVP), auf deutsch Volksbefreiungsfront, eine marxistisch orientierte, politische Organisation, die in der Vergangenheit hinter den zweimaligen sinhalesischen Jugendaufständen stand (RösEL 1997), ein erstaunliches politisches come back verzeichnen. Dieses come back zeichnet sich zwar bislang innerhalb der demokratischen Strukturen ab, kann dennoch aber als ein deutlicher Indikator für die anhaltende Unzufriedenheit der ländlichen Jugendlichen mit dem bestehenden System angesehen werden. 
Entwicklungsanstrengungen, denen es nicht gelingt, Jugendliche in sinnvoller Weise sozial und ökonomisch in die Gesellschaft zu integrieren, laufen Gefahr, die Unzufriedenheit der jungen Generation mit den herrschenden Verhältnissen erneut zu verstärken. Dies bezieht sich nicht nur auf den Süden Sri Lankas, sondern auch auf den vom Bürgerkrieg betroffenen Norden und Osten des Landes, wo vor allem tamilische Jugendliche exponiert sind, sich der LTTE anzuschließen, wenn ihnen adäquate Lebenschancen verwehrt bleiben.

Um realitätsgerechte politische und regionalplanerische Antworten geben zu können, muß die Formulierung geeigneter Maßnahmen zur Gegensteuerung gewalttätiger Konflikte auf vertieften empirischen Kenntnissen über die Bedürfnisse und Nöte Jugendlicher in Sri Lanka aufbauen. Um dafür einen geeigneten Analyserahmen zur Verfügung zu stellen, wird im folgenden eine Erweiterung des Verwundbarkeitsansatzes um konflikttheoretische Dimensionen vorgestellt.

\section{Verwundbarkeit und Konfliktanalyse}

In den letzten Jahren ist es zu einer zunehmenden Differenzierung des Armutskonzeptes gekommen, die weit über die ursprünglich auf reinen Einkommenskriterien beruhenden Ansätze hinausgehen (BAULCH 1996, Bohle 2001, Chambers 1989, Sen 2000). Im Gegensatz zu den auf rein ökonomischen Kriterien beruhenden Armutskonzepten ist als Hauptursache von Verwundbarkeit der Verlust von Handlungsspielräumen sowie eine Destabilisierung von Sozial- und Produktionssicherung anzusehen. Die Stärke dieses Ansatzes liegt dabei vor allem in der differenzierten Wahrnehmung der Struktur von Armut. So beziehen sich die herkömmlichen Konzepte vor allem auf einen Zustand (z.B. geringes Einkommen), wohingegen Verwundbarkeit die Analyse von Armut als einen Prozeß ermöglicht.

Das Verwundbarkeitskonzept hat bislang vor allem in Bezug auf Überlebenssicherungsfragen Anwendung gefunden, und dies vornehmlich im Zusammenhang mit den sozialen Folgen von Umweltkatastrophen sowie der Ernährungssicherung marginaler Bevölkerungsgruppen (BOHLE 2001, CUTTER 2001). Für eine Anwendung der grundlegenden Konzeption des Verwundbarkeitsansatzes für eine Abschätzung gesellschaftlicher Konfliktpotentiale, geht es vor allem um ein vertieftes Verständnis der verschiedenen, konfliktsteigernden Dimensionen gesellschaftlicher Risiken in politischen, ökonomischen, ökologischen sowie soziokulturellen Sphären.

Dafür ist vor allem das Verhältnis zwischen Grund- bedürfnisbefriedigung und Konflikten im Zusammenhang mit sozialem Wandel von Bedeutung (BURTON 1990). Bedürfnisse hinsichtlich Sicherheit, Wohlfahrt, Identität und Freiheit können dabei immer mehr in Widerspruch geraten mit sich schnell wandelnden Gesellschaftsstrukturen:

"The gap between what the individual has in him to become and what society allows him to become increases. (...) Since society changes rapidly, the sense of what is necessary for a satisfactory life becomes less clear. It encourages the aspiration to be highly mobile but difficulties encountered in reaching it create yet another aspiration: to change the structure, create new opportunities and new ways of life» (Roy 1990: 131).

Das ist insbesondere für Jugendliche von Bedeutung, die sich selber noch in einem Prozeß der gesellschaftlichen Positionierung befinden. Auch für den Kontext von Sri Lanka macht es Sinn, der Betrachtung von Bedürfnissen eine besondere Beachtung zukommen zu lassen. Insbesondere vor dem Hintergrund progressiver staatlicher Wohlfahrtsprogramme stellt sich die Frage, in welchen Bereichen Bedürfnisse durch die staatlichen Politiken nicht abgedeckt werden bzw. warum es dennoch zu solchen gewalttätigen Protestreaktionen gegen das System kommen kann, wie sie in Sri Lanka zu beobachten sind.

\section{Geographische Ansätze}

Innerhalb der Geographie sind grundbedürfnisorientierte Fragestellungen vor allem innerhalb der Welfare Geography aufgegriffen worden. Fragen der sozialen Gerechtigkeit und Grundbedürfnisbefriedigung wurden vor allem durch CoATEs et al. (1977) sowie SMITH (1977) in die Geographie eingebracht. Eine gute Übersichtsdarstellung über die (Welfare Geography) als Ansatz zur Beschreibung gesellschaftlicher und sozialräumlicher Ungleichheiten gibt ScHmidT-WULFFEN (1980). Arbeiten zur «Welfare Geography finden sich z.B. bei Bohle (1984), Eyles (1987) oder KNox (1989). Neuere Überlegungen zur Relevanz der «Welfare Geographys stammen von SELL (1994).

Gegenstand der (Welfare Geography) ist die Frage nach der Lebensqualität und den Lebenschancen für bestimmte soziale Gruppen, wobei insbesondere räumlich wirksame Strukturen, Prozesse und Mechanismen untersucht werden, die zu einer Ungleichheit in der Verteilung von Gütern und Dienstleistungen innerhalb einer Gesellschaft führen. Die «Welfare Geography> definiert sich dabei über die Leitfrage: Wer (soziale Perspektive) erhält was (Forschungsgegenstand), wo (geographische Perspektive) und wie (gesellschaftliche Mechanismen). Die Erkenntnis, dass der Ausgleich räumlicher Disparitäten zwar Symptome ver- 
bessern mag, nicht aber die Ursachen von Ungleichheit beseitigen kann, stellt dann die Frage in den Mittelpunkt, wer etwas nicht erhält und warum nicht (BOHLE 1984: 205f.).

Interessante konzeptionelle Anregungen können auch aus der Politischen Geographie gewonnen werden, die sich in den letzten Jahren sehr stark einer räumlichen Konfliktforschung zugewandt hat (z.B. Reuber 2000, REUBER \& WolKERSDORFER 2001). Dabei stehen weniger geographische Fakten im Vordergrund, als vielmehr der räumliche Diskurs politischer Akteure und die Artikulationsformen territorialer Interessen im militärischen, ökonomischen und kulturellen Bereich (REYNOLDS 1994), und die "Art und Weise, wie handlungsrelevante Territorien bestimmt werden" (Ossenbrügge \& SANDNer 1994: 683).

Neben Fragen der Globalisierung und deren Auswirkung auf den Nationalstaat (DodDs 1998, TuATHAIL 1998) rückt dabei auch die Untersuchung sozialer Bewegungen im Zusammenhang mit anti-systemischen Aufständen, das Verhältnis zwischen Staat und Zivilgesellschaft bis hin zu Fragen der Identitätsbildung im Zuge politisch-ökonomischer Transformationsprozesse immer stärker in den Blickwinkel der Forschung (KodRas 1999: 77). Dem traditionellen «Grenzen»Konzept der politischen Geographie wird damit über die ursprünglich rein national-staatliche Definition hinaus eine neue Bedeutung verliehen, die sich vor allem der Konstruktion von sozial-räumlichen Identitäten innerhalb politischer Grenzen (NEwMAN \& PAASI 1998) sowie den Ursachen gesellschaftlicher Gewalt (WATTS 2000) zuwendet.

\section{Verwundbarkeit und das Konzept der Lebenschancen}

Um das Verwundbarkeitskonzept für eine Einschätzung gesellschaftlicher Risiken in Hinblick auf gewaltsame Konfliktpotentiale verwenden zu können, wird in erster Linie eine konzeptionelle Verschiebung des Untersuchungsgegenstandes vorgeschlagen: im Mittelpunkt der Analyse sollen weniger Fragen nach einer «Risiko-Ausgesetztheit» und den verschiedenen Strategien der Überlebenssicherung stehen, sondern die Determinanten für einen adäquaten Zugang zu Lebenschancen. Das Konzept der Lebenschancen möchte sich dabei über Fragen der (Über-) Lebenssicherung hinaus stärker den Widersprüchen zuwenden, die in dem Spannungsfeld zwischen gesellschaftlichen Erwartungshaltungen, sozialen Ambitionen und Bedürfnissen sowie den zur Verfügung stehenden Möglichkeiten, diese Lebensvorstellungen und -ideale umzusetzten, begründet liegen.

Das Konzept der Lebenschancen soll dabei vor allem Hinweise ermöglichen, ob es innerhalb bestimmter gesellschaftlicher Gruppen zu einer $\mathrm{Zu}$ - oder Abnahme von sozialem Konfliktpotential kommt und welche Ursachen für eine Verschärfung oder Reduzierung dieser Konfliktlagen verantwortlich sind. Das Konfliktverständnis liegt hierbei auf der gewaltsamen, destruktiven Konfliktaustragung. Grundsätzlich können Konflikte aber auch als ein positives Potential für gesellschaftliche Veränderungsprozesse angesehen werden, sofern sich die Austragung gewaltfrei und konstruktiv vollzieht.

Diese «Umformulierung» baut auf der grundsätzlichen These auf, daß die Ursachen von sozialen Konflikten zwar in engem Bezug zu Problemen der Überlebenssicherung stehen, häufig aber auch Dimensionen aufweisen, die nicht nur im Zusammenhang mit ökonomischen und sozialen «Risiken» zu sehen sind, sondern auch mit Fragen der Identitätsbildung, der allgemeinen gesellschaftlichen Integration sowie einer politischen und sozio-kulturellen Partizipation zusammenhängen.

Damit wird das Verständnis von Verwundbarkeit in einem Sinne erweitert, wie es vor allem in den jüngsten Ausführungen von Amartya SEN und seiner Definition von «Entwicklung als Freiheit» zum Ausdruck kommt. SEN hat diese individuellen, menschlichen Freiheiten in bezug gesetzt mit ökonomischen Möglichkeiten, politischen Rechten, sozialen Einrichtungen, staatlicher Transparenz und sozialer Sicherheit (SEN 2000: 10). Wichtiger in diesem Zusammenhang sind allerdings SEN's Ausführungen zu der Frage, wie Menschen sich diese Freiheiten nutzbar machen können bzw. welche Voraussetzungen gegeben sein müssen, daß Menschen ihre Grundrechte und Bedürfnisse einfordern können. Dies ist auch der Tenor des jüngsten Human Development Reports, der sich ausführlich mit den Interpretationen zwischen menschlichen Freiheiten und menschlicher Entwicklung auseinandersetzt (UNDP 2000).

Von Bedeutung ist dabei insbesondere der von SEN eingeführte "capability»-Begriff, der viele Parallelen zur Grundbedürfnisdiskussion aufweist, die ebenfalls die Frage nach der Lebensqualität in den Mittelpunkt der Betrachtung stellt (und nicht nur nach der Verfügbarkeit von Einkommen oder Ressourcen fragt) (SEN 2000: 24). SEN legt allerdings eine sehr viel stärkere Betonung auf das Ausmaß individueller Wahlmöglichkeiten zwischen verschiedenen Lebensentwürfen und -stilen (die sog. freedom of choice to achieve various lifestyles - SEN 2000: 75). Die Grundlagen zu seinem Ansatz «Development as Capability Expansion» formulierte SEN bereits 1990:

«Capability reflects a person's freedom to choose between different ways of living. (...) The things people value doing or being can be quite diverse, and the valuable capabilities 
vary from such elementary freedoms as being free from hunger to such complex abilities as achieving self-respect and social participation» (SEN 1990: 44f.).

Gesellschaftliche Verwundbarkeit kann in diesem Verständnis dann vor allem als ein Unvermögen angesehen werden, zwischen alternativen Lebensmöglichkeiten $\mathrm{zu}$ wählen (aufgrund sozialer oder persönlicher Beschränkungen). Als die wichtigsten Förderungsfaktoren zur Erhöhung der Wahlmöglichkeiten betrachten Drèze und SeN (1996: 11f.) den ungehinderten Zugang zu Grundbildung und Gesundheitsdiensten. Allerdings bleibt festzuhalten, daß diese Faktoren nur im Zusammenspiel mit anderen Rahmenbedingungen eine positive Wirkung erzielen können. Die Potentiale, über die jemand durch eine stabile Gesundheit und eine gute Bildung verfügt, müssen sich in der gesellschaftlichen Realität nicht immer auch nutzen lassen: «The political economy of actual use can be very different from the potential possibilities generated» (DRÈZE \& SEN 1996: 6).

Für eine Erfassung der Lebenschancen Jugendlicher aus einer marginalen ländlichen Region in Sri Lanka dienen sechs analytische Dimensionen, die im folgenden zwar getrennt aufgeführt werden, die jedoch auf das Engste miteinander verknüpft sind (siehe Abb. 1). Die Dimensionen der «Umwelt», «Wirtschaft» und «Politik» behandeln vor allem Außenfaktoren, welche die Jugendlichen in ihren Handlungsspielräumen und damit ihren Lebenschancen positiv oder negativ beeinflußen können. Diese Dimensionen decken unter Bezugnahme auf das Verwundbarkeitskonzept vor allem die äußere Seite eventueller Einschränkungen oder Erweiterungen von Lebenschancen ab.

Demgegenüber werden für die Betrachtung der individuellen Potentiale, sich gegen solche Restriktionen seiner Lebenschancen zur Wehr zu setzen oder sogar aktiv die eigenen Wahlmöglichkeiten zu erhöhen, ebenfalls drei analytische Dimensionen herangezogen: die Dimension der «Regionalen Identität», der «Sozialen Integration» sowie des «Empowerment». Die Untersuchung setzt hier auf der individuellen Ebene der Einbindung in lokale, sozio-kulturelle Verhältnisse und dem Ausmaß an potentiellen Initiativkräften zur Verbesserung der eigenen Lebenssituation an. In methodischer Hinsicht ist es dabei für eine Untersuchung von Lebenschancen bestimmter sozialer Gruppen unabdingbar, die subjektiven Wahrnehmungen und Wirklichkeitsinterpretationen der jeweiligen Akteure zu erfassen.

Für das Fallbeispiel der sri lankischen Jugendlichen soll die äußere Seite des Analyserahmens in erster Linie die strukturellen Zwänge bzw. Chancen aufzeigen, welche die Wahlmöglichkeiten, und damit das Ausmaß an Lebenschancen limitieren oder erweitern können. Hier ist schwerpunktmäßig dann vor allem die Formulierung einer geeigneten Entwicklungsplanung und Politik angesprochen, solche strukturellen Zwänge abzumildern bzw. mehr Chancen für bestimmte Gruppen in bestimmten Regionen zu eröffnen. Die innere Seite behandelt die Potentiale (oder Limitierungen), sich gegen strukturelle Benachteiligungen zur Wehr zu setzen bzw. selbstständig alternative Handlungsmöglichkeiten aufzutun. Neben Fragen der Selbsthilfeförderung kann die Analyse hier auch Hinweise bezüglich der Umsetzung von Planungsmaßnahmen liefern.

Das gesellschaftliche Konfliktpotential lässt sich dann am Ausmaß zur Verfügung stehender Lebenschancen festmachen. Je limitierter der «Raum an Lebenschancen" ausfällt, insbesondere bei einem hohen $\mathrm{Maß}$ an strukturellen Einschränkungen, bei gleichzeitig fehlenden lokalen "Widerstandspotentialen», desto größer die Konflikttendenz.

\section{Empirisches Fallbeispiel}

Die obigen Ausführungen sollen im folgenden anhand einiger beispielhafter Ergebnisse zu den Lebenschancen Jugendlicher aus dem in der ländlichen Trockenzone im Süden Sri Lankas gelegenen Hambantota Distrikt verdeutlicht werden. Als Teil eines größeren Forschungsprojektes zu Jugend und Konflikten in Sri Lanka (MAYER 2002) konnten von Mitte 1998 bis Anfang 1999 insgesamt 90, überwiegend qualitative Interviews mit Jugendlichen zwischen 15-29 Jahren aus sieben verschiedenen Dörfern im Hambantota Distrikt durchgeführt werden. Der nur dünn besiedelte Distrikt ist lokalisiert in der südöstlichen Trockenzone der Sri Lankischen Südprovinz (Abb. 2). Der Distrikt ist nur mit knappen Naturressourcen ausgestattet; insbesondere herrscht chronischer Wassermangel. Gleichzeitig besteht eine starke Abhängigkeit von der Landwirtschaft, da kaum alternative Beschäftigungsmöglichkeiten vorhanden sind. Entsprechend wird der Distrikt seit den 70er Jahren als besonders armutsbetroffene Region geführt.

Unter der United National Party (UNP)-Regierung wurden in den 80er Jahren größere Bewässerungsprojekte initiiert (wie z.B. das Lunugamwewa-Projekt), die allerdings aufgrund von Fehlplanungen und Missmanagement nicht die Erwartungen erfüllen konnten, die in sie gesetzt wurden. Darüber hinaus weist der Distrikt auch nach neusten Erhebungen mit die höchste Rate an Jugendarbeitslosigkeit auf (CASS/ SAI 2000).

Der durch naturräumliche und wirtschaftliche Mar- 
ginalität gekennzeichnete Hambantota-Distrikt war vor allem Ende der 80er Jahre auch ein Zentrum der JVP-Aufstände und erlebte damit in der Vergangenheit das höchste Ausmaß an Gewalt im gesamten Land (Peiris 1996, Perera 1999). Auch heute - nach Rückkehr der JVP in das demokratische System - gilt der Distrikt unverändert als Hochburg der JVP.
Das hohe Armutsniveau und Konfliktpotential bei gleichzeitiger Fokussierung neuer entwicklungsplanerischer Maßnahmen auf den Hambantota Distrikt machen die Region überaus geeignet für eine Untersuchung darüber, welche Voraussetzungen eine Regionalentwicklungsplanung erfüllen muß, um den Bedürfnissen jugendlicher Zielgruppen gerecht

\section{«AUSGESETZTHEIT»}

(Exposure)

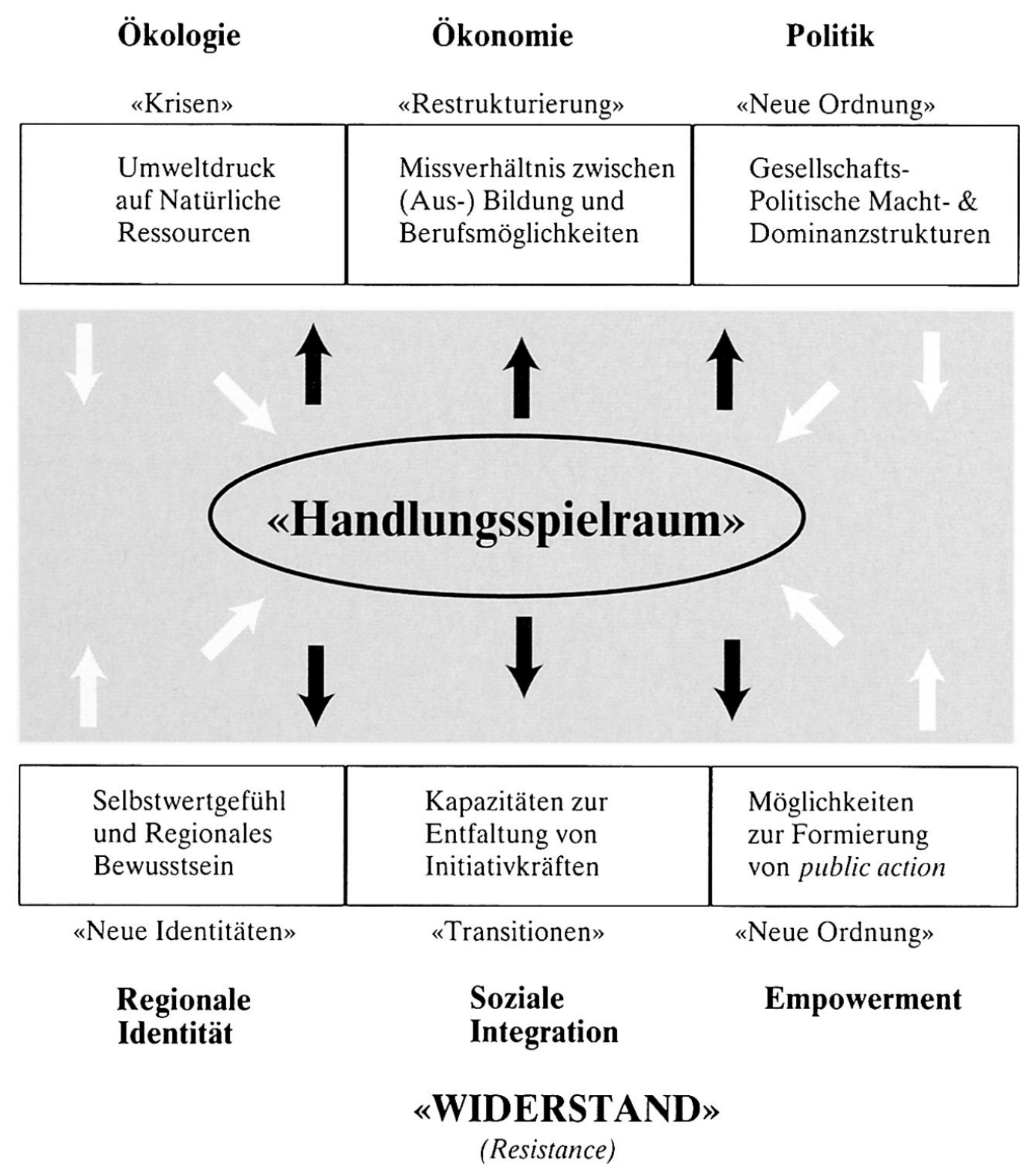

Potentielle

Restriktionen/

Erweiterungen

(Externe Seite)
Potentielle

Restriktionen/

Erweiterungen

(Interne Seite)

Abb. 1: Konzeptioneller Rahmen für die Analyse von Lebenschancen

Conceptual framework for the analysis of livelihood security

Cadre conceptionnel pour l'analyse des chances de vie

Quelle: basierend auf Konzepten von Baulch 1996, Bohle 1998, Chambers 1989, Friedmann 1992, Johnston, TAYlor \& Watts 1995, Sen 2000, WatTs \& Bohle 1993; Entwurf: M. MaYeR 


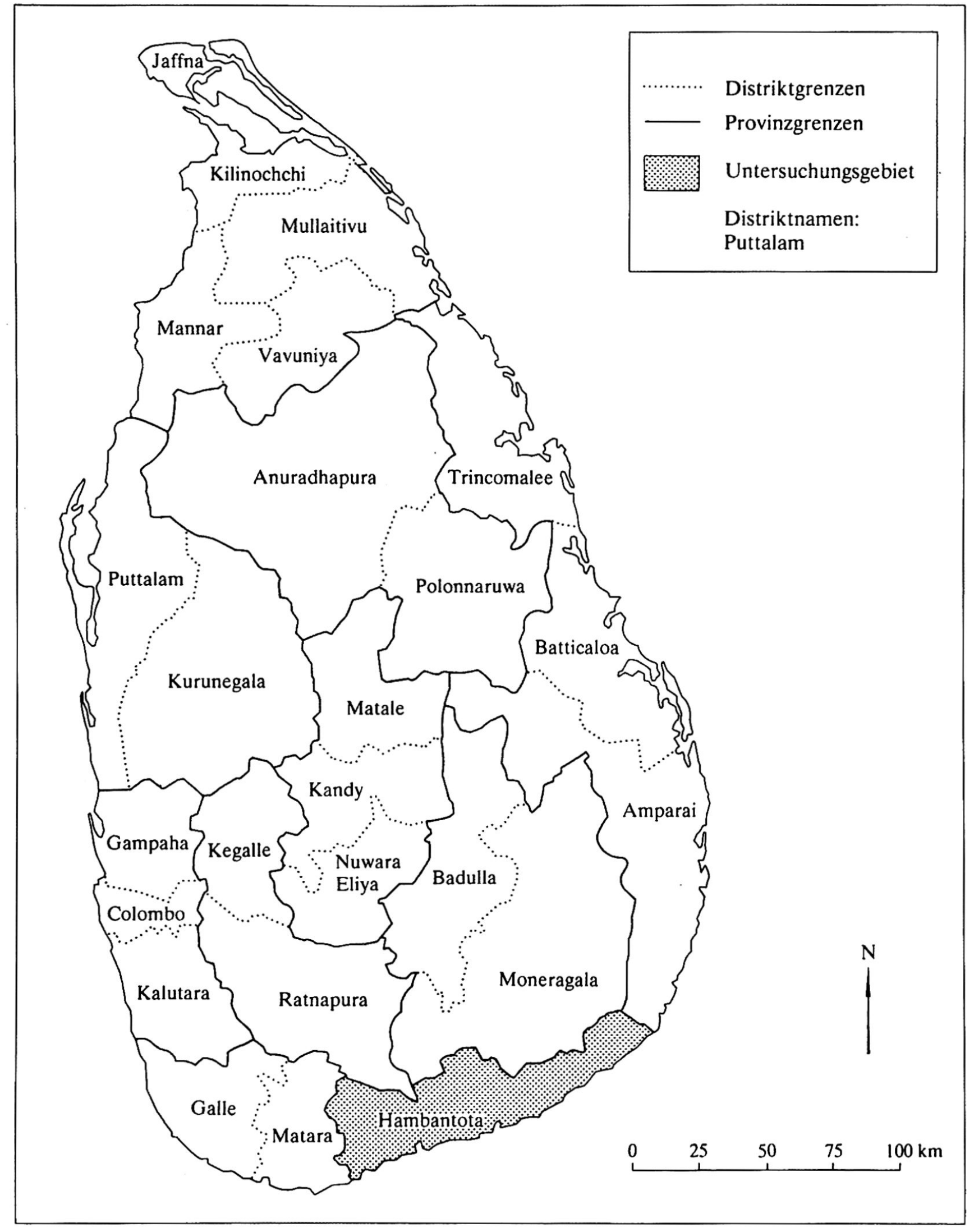

Abb 2: Lage des Untersuchungsgebietes 
zu werden und künftige Konfliktlagen abzumildern.

Im folgenden werden die Aussagen der Jugendlichen unter Anwendung des entwickelten theoretischen Analyserahmens entsprechend um die identifizierten sechs Dimensionen gruppiert, die Einfluss auf die Ausgestaltung der Lebenschancen Jugendlicher haben können. Die Ausführungen beziehen sich - sofern nicht anders vermerkt - auf weibliche und männliche Jugendliche gleichermaßen, da deren Wahrnehmung in vielerlei Hinsicht sehr ähnlich ist und eine Differenzierung in den meisten Fällen nicht nötig erscheint. Wichtigere Kategorien als das Geschlecht scheinen im Kontext von Sri Lanka für die männlichen wie weiblichen Jugendlichen die Identifizierung als Jugendliche an sich sowie die regionale Herkunft zu sein.

\section{Die Politisierung alltäglicher Lebenschancen (Politi- sche Sphäre)}

Die Jugendlichen sehen die Ursachen für ihre Probleme, und hier in ganz besonderem Maße ihren mangelnden Zugang zu akzeptablen Beschäftigungsformen, in der einseitigen Bevorzugung bestimmter Gruppen durch die politische Elite in Colombo. Entscheidend ist hierbei weniger, in welchem Ausma $\beta$ diese Diskriminierung tatsächlich stattfindet, als vielmehr die Tatsache, daß die Verantwortung für die Lösung der eigenen Probleme von den befragten Jugendlichen in hohem Maße bei der politischen Elite gesehen wird. Je weniger dann von dieser Seite eine Lösung der Probleme erfolgt, umso geringer ist deren Akzeptanz und umso mehr wächst die Frustration und anti-staatliche Tendenz unter den Jugendlichen.

"If a young person needs a job today, he or she has to go after a politician. People have been accustomed in a way that they are shamelessly willing to do something like that. The fact that they have been either deprived of employment or that they are largely underemployed, aggravate this situation. When youth see social injustice, they tend to revolt. The LTTE, the JVP and the riots in the universities are revolutions against not only ideology but also the political system itself.» (Hambantota youth, male, $23 \mathrm{yrs}$, 3 rd year undergraduate)

Es zeigt sich sehr deutlich, daß die Jugendlichen in großer Mehrheit von den politischen Verhältnissen enttäuscht sind und eine ausgeprochen negative Meinung über die gewählten Politiker in Sri Lanka haben. Dies bestätigt sich auch durch jüngste Meinungsumfragen: knapp $80 \%$ aller Jugendlichen im Alter von 15-29 haben keine hohe Meinung von der politischen Führung in Sri Lanka (CASS/SAI 2000). Auf der anderen Seite sind sich die Jugendlichen aber sehr stark ihrer Abhängigkeit von eben jenen Politikern bewußt:
«If one can call the ruling party a caste possibly a caste could be benefiting more from the country's development activities (...). The resources are divided up according to the political party that is in power. When a successive government takes over, immediately the workers in the opposition lose their jobs and the ruling party supporters replace them. The Gramasevaka (local government officer) decides who gets the monthly Samurdhi payment (welfare payment for the poor in Sri Lanka). He helps those who help him.» (Hambantota youth, 18 yrs, O-Level educated. male)

Das «Überleben» der Politiker und Staatsangestellten auf regionaler und lokaler Ebenen hängt in starkem $\mathrm{Maße}$ von deren Fähigkeiten $a b$, die verschiedenen Ressourcen, wie z.B. Beschäftigung, unter den jeweiligen Helfern und Parteigängern zu verteilen. Auch wenn die Verteilung von Ressourcen grundsätzlich in der Verantwortung lokaler Politiker liegt, führt deren hohe Abhängigkeit von den Parlamentsabgeordneten und Ministern in Colombo dazu, daß sie deren Vorgaben Folge zu leisten haben. Diese Dominanz der zentralen politischen Ebene wird noch dadurch verstärkt, dass für die lokale Bevölkerung außerhalb der nur alle sechs Jahre stattfindenden allgemeinen Parlamentswahlen keinerlei Mechanismen oder Strukturen bestehen, auf diese Entscheidungsprozesse Einfluss zu nehmen, geschweige denn an ihnen partizipieren zu können.

Insbesondere durch die Politisierung des Arbeitsmarktes sehen sich Jugendliche, die in eine gute Bildung investiert haben, systematisch in ihren Lebenschancen diskriminiert. Vor allem unter arbeitslosen Jugendlichen verstärkt sich dadurch das Gefühl sozialer Ungerechtigkeit mehr und mehr. Unter den interviewten Jugendlichen ist es vor allem diese Politisierung ihrer Lebenschancen, die am meisten zu einer erhöhten Bereitschaft zur Gewaltanwendung zur Durchsetzung der eigenen Interessen führt:

"When have youth gained their demands easily? Everyone has acquired his or her demands through protesting. Nowadays, this also does not work; only threatening one's life will work in order to pursue demands and necessities. I also know that conflicts are not favorable in the event of pursuing one's interests but what are the instances where people have succeeded in pursuing their interests without violence? Since so many years there is no positive sign of acquiring our needs. Therefore, one has to take violent ways even reluctantly." (Hambantota youth, 27 yrs, A-Level educated, male)

\section{Ungeeignete ökonomische Strukturen (Ökonomische Sphäre)}

Der Zugang zu adäquater Beschäftigung gestaltet sich für Jugendliche aus dem ländlichen Süden aus zwei 


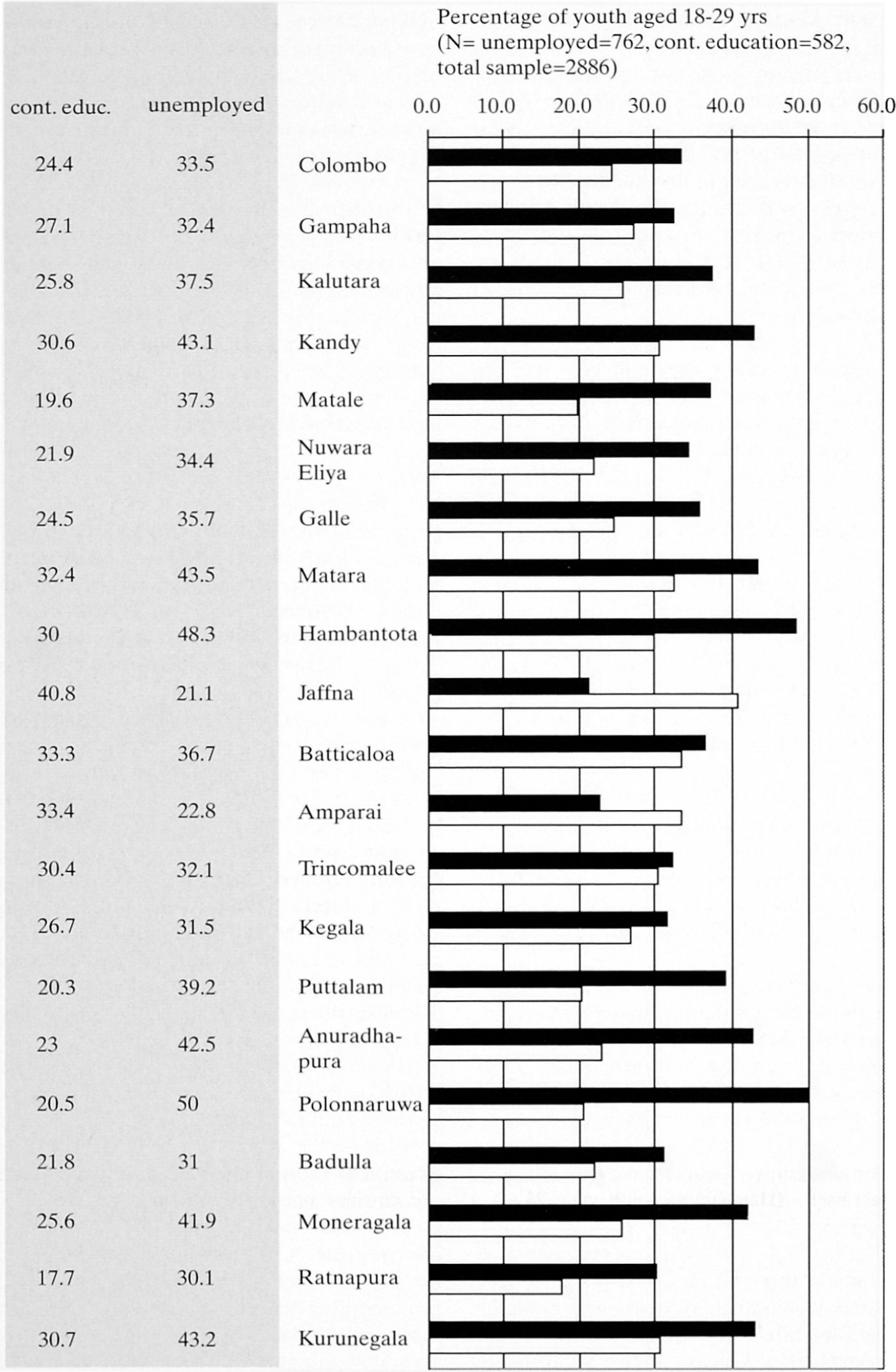

Abb. 3: Jugendliche in Sri Lanka ohne Beschäftigung Unemployed youth in Sri Lanka

Jeunes sans travail à Sri Lanka

Quelle: National Youth Survey Sri Lanka (CASS/SAI 2000) 
Gründen als sehr schwierig: Zum einen passen Ausbildung und Berufsmöglichkeiten nicht zusammen. Das sri lankische Schulsystem bereitet im wesentlichen auf eine nur einer kleinen Minderheit offenstehende Universitätslaufbahn vor und ist wenig anwendungsbezogen für Tätigkeiten außerhalb der staatlichen Administration. In ländlichen Regionen werden weder privatwirtschaftliche noch selbstständige Beschäftigungsformen ausreichend gefördert, noch wird ein ernsthafter Reformversuch unternommen, Jugendliche über die Schulbildung besser auf solche Tätigkeiten vorzubereiten.

Die ländliche Ökonomie bietet Jugendlichen mit einer höheren Bildung immer weniger Optionen im Hinblick auf einen guten bzw. «respektablen» Job. Daraus resultiert die starke Orientierung auf den staatlichen Sektor, der deshalb immer noch vielen Jugendlichen als die einzige Alternative zu einer stagnierenden Landwirtschaft erscheint. Im Kontext der lokalen gesellschaftlichen Wahrnehmungen wird deutlich, daß ein statusbezogener Beruf, mit dem sich soziale und politische Netzwerke zur Überlebenssicherung aufbauen lassen, mehr zählt als rein materielle Beschäftigungskriterien. Eine gesellschaftlich akzeptable Tätigkeit stellt zudem eine wichtige Voraussetzung zur Heirat dar, was in Sri Lanka gleichzeitig als der wichtigste Schritt für den Übergang von der Jugend zum Erwachsensein angesehen wird.

«Sri Lankan culture is such that it does not view selfemployment as employment. It should not be like that. In marriage, the male is required to have a stable job in the private or public sector, preferably with a pension. Selfemployment does not count very much to people.» (Hamabantota youth, $22 \mathrm{yrs}, \mathrm{A} / \mathrm{L}$ educated, male)

Als Konsequenz verweilt ein immer grösserer Anteil von Jugendlichen in einer Situation, in der sie sich entweder als arbeitssuchend empfinden (und dies in ihrer Selbstperzeption dann zumeist als «arbeitslos» angeben) oder einer weiteren Ausbildung nachgehen (vgl. Abb. 3).

\section{Umweltdruck auf knappe Ressourcen (Ökologische Sphäre)}

Zu der von den Jugendlichen wahrgenommenen politischen Diskriminierung und den ihre Erwartungen enttäuschenden wirtschaftlichen Strukturen kommen die immer größeren Restriktionen in der Landwirtschaft aufgrund der abnehmenden Ressourcenbasis, insbesondere in bezug auf Land- und Wasserverfügbarkeit, hinzu. Der Planungsdiskurs, mit seiner starken Ausrichtung auf die Förderung von nicht-landwirtschaftlicher Beschäftigung, richtet sich dabei vor allem an die politisch als «aufrührerisch» erkannte Gruppe der Jugendlichen mit einer sehr guten Schul- bildung. Neben dem Problem, dass diese Planung bislang eher theoretischer Natur geblieben ist und kaum über einfache Selbstbeschäftigungstätigkeiten hinausgehende Arbeitsplätze geschaffen werden konnten, stellt sich zudem die Frage nach der grundsätzlichen Eignung solcher Planungen.

Ein großer Teil der Jugendlichen in den ländlichen Gebieten verfügt lediglich über eine Basisschulbildung und wäre - sofern sich damit der Lebensunterhalt bestreiten lässt - nach wie vor an einer Tätigkeit im landwirtschaftlichen Sektor interessiert, insbesondere wenn sich damit der statusmäßig angesehene Besitz von einem Stück Land verbindet. Über Maßnahmen der Agrarmodernisierung oder geeigneter Landreformen, die den Umweltdruck auf die knappen Ressourcen verringern könnten, wird auf der Planungsebene aber nur wenig reflektiert.

Hinzu kommt, daß die knappen natürlichen Ressourcen, insbesondere Wasser, ebenfalls einer politisch beeinflußten ungerechten Verteilung unterliegen (Bohle, BütTner, Dillen \& Mayer 2000). Dadurch verschärft sich nicht nur der Wettbewerb unter Jugendlichen um Beschäftigungsmöglichkeiten außerhalb der Landwirtschaft, sondern es verstärkt sich auch die Wahrnehmung der Jugendlichen, da $ß$ ihnen Lebenschancen vor allem durch das politische System vorenthalten werden. Ökologische Krisen, wie z.B. Dürren, die in regelmäßigen Abständen die Trockenzonenregionen von Sri Lanka bedrohen, tragen dann nicht nur zu einer Erhöhung von Armut in diesen Regionen bei, sondern können als Folge auch zu einer weiteren Radikalisierung ländlicher Jugendlicher führen. Diese Jugendlichen, die in der Landwirtschaft immer weniger Existenzmöglichkeiten sehen, gleichzeitig aber kaum berufliche Chancen außerhalb dieses Sektors haben, gehören schließlich der spezifischen sozialen Schicht an, die in der Vergangenheit die verschiedenen Jugendaufstände zwar nicht ausgelöst, aber weitgehend getragen haben.

\section{Räumliche Orientierung auf das direkte Umfeld (Sphäre der regionalen Identität)}

Die Jugendlichen zeigen mehrheitlich eine sehr starke Fixierung auf ihr ländliches Umfeld und grenzen sich deutlich von städtischen Lebensstilen und -normen ab. Diese Ablehnung kann sowohl damit zusammenhängen, daß sich viele der Jugendlichen nicht zutrauen, mit einer urbanen Jugend erfolgreich um bessere Arbeitsplätze zu konkurrieren. Zudem empfinden sie sich aufgrund ihrer regionalen Herkunft per se in einer untergeordneten Position, insbesondere im Falle kurzer Arbeitserfahrungen in Colombo. 
Auf der anderen Seite gilt es aufgrund traditionellen Statusdenkens als sozial inadäquat, ab einem gewissen Bildungsniveau bestimmte «niedrige» Tätigkeiten anzunehmen, die außerhalb der eigenen Dorfgemeinschaft für viele Jugendliche durchaus akzeptabel sein könnten. Diese Jugendlichen befinden sich also in einer Situation, in der weder das Verbleiben im Dorf noch die Migration nach Colombo möglich erscheinen und sich sowohl in Bezug auf ihr Befinden als Jugendliche (vor dem Hintergrund der allgemeinen Wahrnehmung von Jugend als Problemgruppe) wie auch von ihrer regionalen Herkunft her gesellschaftlich stigmatisiert sehen.

Dabei ist allerdings festzuhalten, $\mathrm{da} ß$ regionale Potentiale für Beschäftigung außerhalb des unmittelbaren dörflichen Kontextes von den Jugendlichen bislang wenig wahrgenommen werden. Die gesellschaftliche Stigmatisierung von Jugendlichen in Sri Lanka läuft außerdem Gefahr, Passivität und Frustration unter Jugendlichen zu verstärken, anstatt ihre Motivation zu fördern, konstruktive Eigeninitiativen zur Verbesserung ihrer Lebensumstände zu starten.

"Youth do a service to society especially before marriage. Young people are brave and face any situation. But society doesn't render much of a service to them and their ideas are not much taken notice of. Youth are viewed as rebellious troublemakers. It is because parents, teachers and politicians stay away from their duties that the capabilities of youth cannot be realized. (...) But young people are a group who wants to live independently and want to achieve a permanent status in life. But in today's context these are mere fantasies. Young people are frustrated and their hopes are shattered, so they want to achieve things through good or bad means. There might be uprisings in the future as well.» (Hambantota youth, $24 \mathrm{yrs}, 2^{\text {nd }}$ year University student, female)

\section{Mangelhafte gesellschaftliche Integration (Sphäre der sozialen Integration)}

Insgesamt ist festzustellen, daß die Jugendlichen kaum in traditionelle Gesellschaftsstrukturen integriert sind. Das bezieht sich sowohl auf staatliche Einrichtungen wie diverse zivilgesellschaftliche Institutionen sowie in gewissen Maße auch auf den familiären Zusammenhalt.

"There are no institutions that support the interests of youth in my village. It is a weakness of the government that they still haven't established institutions that help youth. The monk in the village is suitable to help solving youth problems because he doesn't belong to a political party. People do not take youth problems seriously; they regard them as minor matters that small children encounter.» (Hambantota youth, 27 yrs, A-Level educated, male)
«Society looks at our problems with squint eyes. Even if they see us talking to male friends, they look with squint eyes. They don't rescue people who were fallen into trouble, but they further mentally weaken them. Parents themselves are uneducated and as such they also can't give advice to their children neither do they look after them properly anyway.» (Hambantota youth, 19 yrs, O-Level educated, female)

Das Problem eines fehlenden sozialen Zusammenhaltes verschärft sich vor allem für arbeitslose Jugendliche, denen kaum Möglichkeiten zur Verfügung stehen, über alternative Aktivitäten ein gesellschaftliches Engagement zu zeigen, womit die mit der Arbeitslosigkeit einhergehende gesellschaftliche Stigmatisierung kompensiert werden könnte. Im Kontext der Auflösung traditioneller dörflicher Sozialstrukturen kommt einem respektablen Job hinsichtlich der eigenen Positionierung in den lokalen Gesellschaftstrukturen daher eine immer größere Bedeutung zu.

\section{Fehlende Kapazitäten für Selbsthilfe-Aktivitäten (Sphäre des Empowerments)}

Eine Folge dieser mangelnden gesellschaftlichen Integration Jugendlicher ist die geringe Entwicklung von gruppenbezogenen Aktivitäten in den Dörfern. Das Fehlen von wirklichen «Graswurzel»-Bewegungen und partizipativen gesellschaftlichen Strukturen auf der lokalen Ebene muß als ein wichtiger Grund dafür angesehen werden, daß Interventionen seitens verschiedener Nicht-Regierungsorganisationen sowie Projekten der internationalen Entwicklungszusammenarbeit zur sozialen Mobilisierung und Förderung von Selbsthilfekapazitäten, häufig wenig erfolgreich verlaufen.

«More than 100 NGO's have come to our village; they come and do something useless and leave. Most of them donate spectacles or provide some facilities for fishery. They all say that commonly work is being done. Only their big shots gain commonly.» (Hambantota youth, 27 yrs, A-Level educated, male)

«Don't ask about NGO's. They have consumed the country and now there is nothing useful left in the country. If things go on like this I don't know whose blessings will be there for our people.» (Hambantota youth, $19 \mathrm{yrs}$, O-Level educated, female)

Mit denselben Schwierigkeiten werden sich auch die geplanten staatlichen Regionalentwicklungsprojekte konfrontiert sehen, wenn es nicht gelingt, Jugendliche nicht nur als direkte Zielgruppe anzusprechen, sondern insgesamt zivilgesellschaftliche Strukturen auf der lokalen Ebene zu etablieren und zu konsolidieren. 
"If the villagers have the motivation they will be able to come forward and compel the relevant authorities to do something. But there is no unity among villagers. Because of party politics there are family and neighborhood conflicts; due to party allegiances people hate each other. There are always political differences that are coming forward even for common development activities, so that there is no cooperation at all.» (Hambantota youth, 23 yrs, O-Level educated, male)

Als das wichtigste Mittel für eine Verbesserung der eigenen Lebensumstände wird Bildung angesehen, grundsätzlich ein eher individualistischer Ansatz, der keine positiven Auswirkungen auf die Förderung von Gruppeninitiativen hat. Die Frustration derjenigen, denen der Zugang zu erwünschten Lebenschancen verwehrt bleibt, trägt grundsätzlich zu einer Erhöhung der Gewaltbereitschaft unter den Jugendlichen bei.

"The education only benefits the rich people. The poor, having spent $7-8$ years for a degree have to wait at home requesting a job. No matter how far we study we cannot live a satisfying life in this country. In order for people like me to serve the country and to be appointed for work as we wished to in gaining education, the existing political system has to be wiped out.» (Hambantota youth, $27 \mathrm{yrs}$, A-Level educated, male)

\section{Schlußfolgerungen}

Die Anwendung des Verwundbarkeitskonzeptes als Analyserahmen zur Einschätzung von Konfliktpotential unter ländlichen Jugendlichen in Sri Lanka erbringt deutliche Indikatoren für eine zunehmende Diskriminierung und Benachteiligung Jugendlicher in politischen, ökonomischen wie ökologischen Sphären, bei gleichzeitig geringen Möglichkeiten, sich innerhalb der lokalen Gemeinschaft alternative Lebensperspektiven aufzubauen. Konsequenterweise kommt in vielen Aussagen der interviewten Jugendlichen eine deutliche Zunahme der Bereitschaft zur Gewaltanwendung zu Tage.

Im Hinblick auf die Ausgestaltung ihrer Lebenschancen kann der fehlende Zugang zu geeigneter Beschäftigung als ein zentrales Problem vieler ländlicher Jugendlicher angesehen werden. Im Kontext von Sri Lanka geht es dabei allerdings weniger um Fragen der Überlebenssicherung, sondern vielmehr um Fragen der sozialen Akzeptanz und des individuellen Statusdenkens der Jugendlichen im Zusammenhang mit verschiedenen Beschäftigungsmöglichkeiten. Die Disparitäten verlaufen in Sri Lanka außerdem entlang einer Stadt-Land-Achse. Eine städtische Bildungselite in Colombo, die eigentlich eine Minderheit im eigenen Land darstellt, kann sich erfolgreich in einen globalen Kontext einbinden. Die ländliche Mehrheit des Landes gerät dagegen in eine marginale Position. Insbesondere unter ländlichen Jugendlichen steigert diese Situation anti-Colombo bzw. anti-kapitalistische Ressentiments.

Die Implementierung einer erfolgreichen Regionalplanung sieht sich neben der passiven EmpfängerMentalität vieler Jugendlicher, die letztlich auch ein Ergebnis der Sozialisierung durch die elterliche wie schulische Erziehung darstellt, ebenfalls dem Einfluß des hochgradig zentralistischen Parteiensystemes in Sri Lanka ausgesetzt, welches bis auf die untersten regionalen Ebenen reicht. Sri Lanka verfügt zwar formal über sehr dezentral ausgeprägte Verwaltungsstrukturen, die jedoch über ein umfangreiches Patronagenetzwerk auf allen Ebenen unter der völligen Kontrolle der politischen Elite in Colombo stehen.

Die verschiedenen Ursachendimensionen der vorgestellten Konfliktanalyse können aber auch dazu dienen, geeignete Strategien zur Konfliktreduzierung anzudenken. In dieser Hinsicht kann als die wichtigste Schlußfolgerung festgehalten werden, daß es zur Vermeidung weiterer Jugendkonflikte in Sri Lanka vor allem darauf ankommt, ein Gegengewicht zur Überdominanz der politischen Elite auf der lokalen Ebene zu schaffen. Dabei muß es vor allem darum gehen, Strukturen aufzubauen, welche die lokale Bevölkerung, und hier insbesondere die junge Generation, in die Lage versetzen, über Ressourcen zu bestimmen, die nicht unter der Kontrolle von Colombo stehen. Der Regionalplanung käme dabei vor allem die Aufgabe zu, die optimale Ressourcenbasis in den jeweiligen Regionen zu identifizieren bzw. zu fördern.

Für die Umsetzung einer solchen demokratischen Dezentralisierung verfügt Sri Lanka über das Potential einer gebildeten und politisch sensiblen Jugend auf der lokalen Ebene. Wenn dieser Jugend die entsprechenden Strukturen zur Verfügung gestellt werden, eine aktive Rolle im Entwicklungsprozeß einzunehmen, kann sich darüber auch das soziale Konfliktpotential in Sri Lanka verringern. Hierzu ist vor allem ein Vergleich mit der «People's Campaign for Decentralized Planning» in Kerala sehr aufschlussreich und relevant für den sri lankischen Kontext (siehe z.B. Heller 1999, IsaAC 2000). Darüber hinaus könnte die Jugend so zum Motor einer neuen politischen Kultur und nachhaltigen Entwicklung in Sri Lanka werden.

\section{Literatur}

Anderson, M.B. (1999): Do No Harm: How Aid Can Support Peace - or War. - Boulder u.a.: Rienner.

ArJuna Consulting Co. LTd. (ed.) (1997): Atlas of Sri Lanka. - Colombo.

Bauer, E., Bigdon, C. \& B. Korf (2000): Anspruch und Wirklichkeit in der Konfliktbearbeitung - Was kann 
Entwicklungszusammenarbeit wirklich leisten? Das Beispiel Sri Lanka. - In: Peripherie 79, 20. Jg.: 43-68.

BAulch, B. (1996): Editorial - The New Poverty Agency: A Disputed Consensus. - IDS Bulletin 27, 1: 1-10.

Bohle, H.-G. (1984): Perspektiven der Welfare Geography. Am Beispiel südindischer Stammesmärkte. In: Hotres, K.H. \& H. Uhlig (Hrsg.): Probleme der Entwicklungsländerforschung in Süd-und Südostasien. - = Materialien zur Raumordnung 27, Bochum.

BoHLE, H.-G. (1998): Geographie und Entwicklungsforschung. Beiträge der Sozialgeographie zum Problemkreis von «Global Change». - In: KarrascH, H. (Hrsg.): Geographie: Tradition und Fortschritt. - Festschrift zum 50-jährigen Bestehen der Heidelberger Geographischen Gesellschaft (= HGG-Journal 12), Heidelberg: 71-86.

BoHLE, H.-G. (2001): Vulnerability and Criticality: Perspectives for Social Geography. - In: Update IHDP 2/01:1-5.

Bohle, H.-G., Bürtner, H., van Dillen, S. \& M. Mayer (2000): Water Conflicts and Environmental Entitlements. Local-Level Experience from South Asia. - In: IGU Bulletin 50, 1: 39-46.

Burton, J. (ed.) (1990): Conflict: Human Needs Theory. - London: Macmillan.

CASS/SAI (Center for Applied Sociological Studies/ South Asia Institute) (2000): Understanding Sri Lankan Youth: Some Selected Aspects and Illustrated Data. National Youth Survey - Overview Report, Colombo. Chambers, R. (1989): Vulnerability, Coping and Policy. - IDS-Bulletin 20.

COATES, B.E. et al. (1977): Geography and Inequality. Oxford, London: Oxford University Press.

CoHEN, P. (1997): Rethinking the Youth Question. Education, Labor and Cultural Studies. - Basingstoke, Hamshire u.a.: Macmillan.

CutTer, S.L. (2001): A Research Agenda for Vulnerability Science and Environmental Hazards. - In: Update IHDP 2/01: 8-9.

DodDs, K. (1998): Political Geography I: The Globalization of World Politics. - In: Progress in Human Geography 22, 4: 595-606.

Drèze, J. \& A. Sen (1996): India. Economic Development and Social Opportunity. - New Delhi u.a.: Oxford University Press.

E $+Z$ (2000): Krisenprävention und Konfliktbearbeitung. - Themenheft, Jg. 41, 3.

EyLes, J. (1987): The Geography of the National Health: an Essay in Welfare Geography. - London: Croom Helm.

FAHRENhorst, B. (2000): Die Rolle der Entwicklungszusammenarbeit in gewalttätigen Konflikten. - SIDBerlin-Berichte 11, Berlin.

FriedmanN, J. (1992): Empowerment. The Politics of Alternative Development. - Cambridge, Mass., Oxford: Blackwell.
Heller, P. (1999): The Labor of Development. Workers and the Transformation of Capitalism in Kerala, India. - Ithaca, NY: Cornell University Press.

IsAAC, T.M.T. (2000): Local Democracy and Development. People's Campaign for Decentralized Planning in Kerala. - New Delhi: Left Word Books.

Johnston, R.J., TAYlor, P.J. \& M.J. WatTs (eds) (1995): Geographies of Global Change: Remapping the World in the Late Twentieth Century. - Oxford: Blackwell.

Knox, P.L. (1989): The Vulnerable, the Disadvantaged, and the Victimized: Who They Are and Where They Live. - In: Herbert, D.T. \& D.M. Smith (eds): Social Problems and the City. - Oxford: Oxford University Press.

Kodras, J.E. (1999): Geographies of Power in Political Geography. - In: Political Geography 18, 99: 75-79.

MARGA (2001): The Cost of the War. The Economic, Socio-Political and Human Cost of the War in Sri Lanka. - National Peace Council, Colombo.

MAYER, M. (2002): Jugendkonflikte und Entwicklungsplanung: Eine sozialgeographische Analyse der Lebenschancen Jugendlicher und der Potentiale dezentraler Planung in Sri Lanka. - Studien zur Geographischen Entwicklungsforschung, Saarbrücken (im Druck).

MAYER, M. (2000): Life Opportunities and Youth Conflict in Sri Lanka: Challenges for Regional Development Planning. - In: Hettige, S.T. \& M. MaYer: Sri Lanka at Crossroads: Dilemmas and Prospects After 50 Years of Independence. - New Delhi: Macmillan: 156-168.

Mehler, A. \& C. Ribaux (2000): Krisenprävention und Konfliktbearbeitung in der TZ. Ein Überblick zur nationalen und internationalen Diskussion. - GTZ, Eschborn.

National Human Development Report (1998): Regional Dimensions of Human Development - Sri Lanka. - Colombo: UNDP.

Newman, D. \& A. PaAsi (1998): Fences and Neighbours in the Postmodern World: Boundary Narratives in Political Geography. - In: Progress in Human Geography 22, 2: 186-207.

Ossenbrügge, J. \& G. Sandner (1994): Zum Status der Politischen Geographie in einer unübersichtlichen Welt. - In: Geographische Rundschau 46, 12: 676-684.

PeIRIS, G.H. (1996): Insurrection and Youth Unrest in Sri Lanka. - Paper presented at the International Workshop on «Causes of Conflict and Conflict Resolution>, 15.-17. July, Kandy.

Perera, S. (1999): Stories of Survivors. Socio-Political Contexts of Female Headed Households in Post-Terror Southern Sri Lanka (Volume I). - New DelhiColombo.

Reuber, P. (2000): Die Politische Geographie als handlungsorientierte und konstruktivistische Teildisziplin - angloamerikanische Theoriekonzepte und aktuelle 
Forschungsfelder. - In: Geographische Zeitschrift 88, 1:36-52.

Reuber, P. \& G. Wolkersdorfer (eds) (2001): Politische Geographie. Handlungsorientierte Ansätze und Critical Geopolitics. - Heidelberger Geographische Arbeiten 114, Heidelberg.

REYNOLDS, D.R. (1994): Political Geography: the Power of Place and the Spatiality of Politics. - In: Progress in Human Geography 18, 2: 234-247.

Roche, J. \& S. TUCKer (1997): Youth in Society. Contemporary Theory, Policy and Practice. - London.

RöSEL, J. (1997): Der Bürgerkrieg auf Sri Lanka: Der Tamilenkonflikt: Aufstieg und Niedergang eines sinhalesischen Staates. - Baden-Baden: Nomos Verlagsgesellschaft.

RorH, R. \& D. Rucht (Hrsg.) (2000): Jugendkulturen, Politik und Protest. Vom Widerstand zum Kommerz? - Opladen: Leske + Budrich.

Roy, R. (1990): Social Conflicts and Needs Theories: Some Observations. - In: Burton, J. (ed.) (1990): Conflict: Human Needs Theory. - London: Macmillan: 125-148.

Rucht, D. \& R. Rотн (2000): Weder Rebellion noch Anpassung: Jugendproteste in der Bundesrepublik 1950-1994. - In: Roth, R. \& D. Rucht (Hrsg.) (2000): Jugendkulturen, Politik und Protest. Vom Widerstand zum Kommerz? - Opladen: Leske + Budrich: 283-304. Rudolph, H.-H. (1997): «Jetzt reden wir!»: Jugend, lebensweltbezogene Bildung und Gemeindeentwicklung in Lateinamerika. - Forschungsreihe: Erziehung und Gesellschaft im internationalen Kontext 12, Frankfurt am Main: Verlag für interkulturelle Kommunikation.

SCHMidT-WulfFen, W. (1980): 'Welfare Geography) oder: Leben in einer ungleichen Welt. Angelsächsische Ansätze zu einem 〈Paradigma〉 gesellschaftlicher und sozialräumlicher Ungleichheiten. - In: Geographische Zeitschrift 68, 2: 107-120.

SelL, S. (1994): Räumliche Sozialpolitik. Möglichkeiten und Grenzen Sozialpolitikwissenschaftlicher Konzepte in der Geographie. - In: Geographische Zeitschrift, 82. Jg., H. 3: 154-165.

SEN, A. (1990): Development as Capability Expansion. - In: Griffin, K. \& J. KNIGHT (eds): Human Development and the International Development Strategy for the 1990s. - Basingstoke u.a.: Macmillan: 41-58.

Sen, A. (2000): Development as Freedom. - New York: Knopf, Borzoi Books.

Seneratne, J.P. (1997): Political Violence in Sri Lanka, 1977-1990: Riots, Insurrections, Counter-Insurgencies, Foreign Intervention. - Amsterdam: VU University Press.

Sмiтh, D. (1977): Human Geography - A Welfare Approach. - London: E. Arnold.

TUathail, G.O. (1998): Political Geography III: Dealing With Deterritorialization. - In: Progress of Human Geography 22, 1: 81-93.
UNDP (United Nations Development Programme) (2000): Human Development Report 2000. - New York: Oxford University Press.

WATTS, M. (2000): Geographies of Violence and the Narcissism of Minor Difference. - In: Hoyler, M. (ed.): Struggles Over Geography: Violence, Freedom and Development at the Millenium. - Hettner-Lecture 1999 with Michael J. WatTs, Department of Geography, University of Heidelberg.

WatTs, M. \& H.-G. Bohle (1993): The Space of Vulnerability: the Casual Structure of Hunger and Famine. In: Progress in Human Geography 17, 1: 43-67.

WYN, J. \& P. DWYER (1999): New Directions in Research on Youth in Transition. - In: Journal of Youth Studies 2, 1:5-21.

WyN, J. \& R. White (1997): Rethinking Youth. London u.a.: Sage Publications.

\section{Zusammenfassung: Jugend, Verwundbarkeit und sozi- ale Diskriminierung. Lebenschancen und Konfliktpo- tentiale ländlicher Jugendlicher in Sri Lanka}

Krisen- und Konfliktprävention gewinnt innerhalb der entwicklungspolitischen Diskussion zunehmend an Bedeutung. Für ein vertieftes Verständnis der Zusammenhänge zwischen dem Bedürfnis nach angemessener Lebenssicherung sowie den konfliktsteigernden Folgen diskriminierender Einschränkungen von Handlungsspielräumen wird eine konzeptionelle Erweiterung des Verwundbarkeitsansatzes vorgestellt. Für eine geographische Konfliktanalyse werden zum einen Anregungen aus der Welfare Geographie und der Politischen Geographie sowie aus neueren Ansätzen von Amartya Sen zur menschlichen Entwicklung als Freiheit entnommen. Zur Bewertung von gesellschaftlichen Konfliktpotentialen wird insbesondere Jugend als eine wichtige Referenzgruppe herangezogen und am Beispiel von Sri Lanka empirisch unterlegt. Interviews mit ländlichen Jugendlichen aus dem Hambantota Distrikt im Süden des Landes, der sowohl von einem hohen $\mathrm{Ma}$ an Armut wie auch Konfliktbereitschaft gekennzeichnet ist, unterstreichen die These, dass weniger Fragen der direkten Überlebenssicherung konfliktfördernd sind, sondern vielmehr Fragen der sozialen Akzeptanz und individueller Lebensvorstellungen. Strategien zur Konfliktreduzierung müssen zum einen Jugendliche als eine direkte Zielgruppe konkreter adressieren, und zum anderen eine Regionalplanung fördern, die einer Politisierung der Ressourcen- und Lebenschancenverteilung auf der lokalen Ebene effektiv entgegenwirkt.

Summary: Youth, Vulnerability and Social Discrimination. Livelihood security and conflict potential of rural youth in Sri Lanka

Crisis and conflict prevention are rapidly gaining currency within political development discourse. In order 
to understand the relations between the need for an adequate livelihood security and the conflict potential of discriminatory restrictions of activity, a conceptual broadening of the vulnerability approach is put forward in this article. The discussion on geographical conflict analysis draws on approaches found in Welfare Geography and Political Geography, as well as on recent concepts by AmarTYA SEN on human development as a human right. The evaluation of conflict potential in society centres on youth as an important reference group and is supported by empirical data from Sri Lanka. Interviews with rural youth in the Hambantota district in southern Sri Lanka, who are not only to a large degree considered poor but open to violence, confirm the theory that it is social acceptance and individual expectations of life that nurture violence rather than questions of survival. Strategies aiming to reduce conflict should thus not only address the youth directly, but should also encourage a regional planning that effectively prevents the distribution of resources and livelihood security being anything more than a political rhetoric.

\section{Résumé: Jeunesse, vulnérabilité et discrimination sociale: chances de vie et potentialités conflictuelles pour jeunes ruraux à Sri Lanka}

La prévention des crises et des conflits prend une signification croissante dans la discussion politique relative au développement. Nous présentons ici un élargissement conceptuel de la notion de vulnérabilité en vue de comprendre de manière plus approfondie les rapports existant entre le besoin d'une sécurisation adaptée de la vie et les répercussions grandissantes des conflits inhérents aux restrictions en matière de discrimination. L'analyse conflictuelle géographique se fonde d'une part sur des apports de la géographie du bien-être et de la géographie politique, d'autre part sur les observations plus récentes d'AmarTYA SEN sur le développement humain au regard de la liberté. L'appréciation des potentialités conflictuelles sociétales est surtout appuyée sur la jeunesse en tant que groupe de référence; le Sri Lanka sert d'exemple empirique. Des interviews effectuées en milieu rural jeune dans le district Hambantota, dans le sud du pays, qui se caractérise à la fois par un taux de pauvreté élevé et de propension conflictuelle renforcent la thèse selon laquelle les questions relatives à la sécurisation directe de la vie suscitent moins de conflits que celles propres à l'acceptation sociale et aux représentations individuelles de l'existence. Les stratégies visant la réduction des conflits doivent d'une part s'adresser de manière plus concrète aux jeunes en tant que groupe directement interpellé, d'autre part promouvoir un aménagement régional susceptible de contrer efficacement à l'échelle locale la politisation de la répartition des ressources et des espérances de vie.

Dr. Markus Mayer, Development Studies Institute, University of Colombo, P.O. Box 1490, Colombo, Sri Lanka.

e-mail: imcap@eureka.lk

Manuskripteingang/received/manuscrit entré le 4.10.2001

Annahme zum Druck/accepted for publication/accepté pour l'impression: 25.2 .2002 